\title{
The Generalized Fock Implementation of Complex Orthogonal Transformations
}

By

\author{
Paul L. RoBinson*
}

\begin{abstract}
We determine precise necessary and sufficient conditions for a complex orthogonal transformation to admit a generalized implementing operator in a Fock representation, extending classical results due to Shale-Stinespring and Carey-Palmer.
\end{abstract}

\section{§0. Introduction}

The Fock representation determined by a compatible complex structure on a real Hilbert space serves as the standard model of a free fermion field and as the foundation for further fermionic models in quantum field theory and quantum statistical mechanics. Regarding this representation as a quantization of the original real inner product space, the problem naturally arises of determining which (classical) orthogonal transformations of the real inner product space are represented by (quantum) unitary operators on the Fock space.

In order to be more explicit, let $V$ be the original real inner product space and $J \in O(V)$ a compatible complex structure: fermionic Fock space $\wedge[V]$ is the completion of the (complex) exterior algebra $\wedge(V)$ relative to a natural inner product; the Fock representation $\pi: V \rightarrow B(\wedge[V])$ of $V$ by self-adjoint bounded linear operators on $\wedge[V]$ is defined in terms of creators (multiplication operators) and annihilators (linear antiderivations). Now, we shall say that the orthogonal transformation $g \in O(V)$ is unitarily implemented in the Fock

Communicated by T. Kawai, December 2, 1999

1991 Mathematics Subject Classification(s): 15A66, 15A75, 81R10, 81S05

* Department of Mathematics, University of Florida, 358 Little Hall, P.O. Box 118105, Gainesville FL, U.S.A. 
representation $\pi$ if there exists a unitary (implementing) operator $U$ on $\wedge[V]$ satisfying

$$
v \in V \Rightarrow U \pi(v)=\pi(g v) U .
$$

A famous theorem of Shale and Stinespring establishes that such a $U$ exists if and only if the antilinear part $A_{g}=\frac{1}{2}(g-J g J)$ is of Hilbert-Schmidt class.

The real inner product on $V$ extends to the complexification $V_{\mathbb{C}}$ to define both a symmetric bilinear form $(\cdot \mid \cdot)$ and a Hermitian inner product $\langle\cdot \mid \cdot\rangle$. The eigenspaces $V^{ \pm}=\operatorname{Ker}\left(J_{\mathbb{C}} \mp i I\right)$ of the complexification $J_{\mathbb{C}}$ yield a decomposition $V_{\mathbb{C}}=V^{+} \oplus V^{-}$into $\langle\cdot \mid \cdot\rangle$-perpendicular $(\cdot \mid \cdot)$-isotropic subspaces, whose corresponding projection operators will be written $P^{+}: V_{\mathbb{C}} \rightarrow V^{+}$and $P^{-}: V_{\mathbb{C}} \rightarrow V^{-}$. Each complex orthogonal transformation $G \in O\left(V_{\mathbb{C}}\right)$ extends naturally to an automorphism of the purely algebraic complex Clifford algebra but need not extend to its enveloping $C^{*}$ algebra; accordingly, operators implementing $G$ in the (complexified) Fock representation need not be unitary and may actually be unbounded. In fact, a theorem of Carey and Palmer exhibits a dense $\pi$-stable complex subspace $\mathcal{D} \subset \wedge[V]$ and assigns to each $G \in O\left(V_{\mathbb{C}}\right)$ for which $P^{+} G P^{-}+P^{-} G P^{+}$is Hilbert-Schmidt and $\left(P^{+} G P^{+}+P^{-} G P^{-}\right)-I$ is trace-class a complex-linear automorphism $U$ of $\mathcal{D}$ such that

$$
v \in V_{\mathbb{C}} \Rightarrow U \pi(v)=\pi(G v) U
$$

It is entirely reasonable to seek a context within which more general complex orthogonal transformations of $V_{\mathbb{C}}$ are suitably implemented in the Fock representation. Here we opt for what is arguably the most flexible context. To be explicit, we consider as fundamental the Fock representation $\pi$ on the exterior algebra $\wedge V$ together with the induced representation $\pi$ on its full (purely algebraic) antidual $\wedge V^{\prime}$ comprising all antilinear functionals $\wedge V \rightarrow \mathbb{C}$. Notice that $\wedge V^{\prime}$ contains as a canonical copy of $\wedge[V]$ the space comprising all bounded antilinear functionals $\wedge V \rightarrow \mathbb{C}$. In this context, we define a generalized Fock implementer for $G \in O\left(V_{\mathbb{C}}\right)$ to be a nonzero complex-linear map $U: \wedge V \rightarrow \wedge V^{\prime}$ that intertwines $\pi$ on $\wedge V$ with $\pi$ on $\wedge V^{\prime}$ in the sense

$$
v \in V_{\mathbb{C}} \Rightarrow U \pi(v)=\pi(G v) U
$$

It is relatively straightforward to see that if $G$ admits a generalized Fock implementer then the compression $P^{-} G \mid V^{-}$has finite-dimensional kernel. Perhaps surprisingly, the converse is true: if the compression $P^{-} G \mid V^{-}$has finitedimensional kernel then $G$ admits generalized Fock implementers. Our proof 
that this is so proceeds by applying successive complex orthogonal reflections to reduce the kernel of $P^{-} G \mid V^{-}$to zero and then exhibiting a specific generalized Fock implementer when $P^{-} G \mid V^{-}$is injective. The flexible purely algebraic nature of this context facilitates adaptation to more restricted contexts upon the imposition of analytic constraints: thus, it permits recovery of the Shale-Stinespring and Carey-Palmer theorems; also, it suggests the possibility of handling further situations in which traditional implementing operators cannot be defined.

The organization of this paper is as follows. "Complex orthogonal transformations" presents essentially those aspects of the complex orthogonal group $O\left(V_{\mathbb{C}}\right)$ that are directly relevant to our approach. "Generalized Fock implementation" develops and establishes the aforementioned necessary and sufficient condition in order for an element of $O\left(V_{\mathbb{C}}\right)$ to admit a generalized Fock implementer. Finally, "Remarks" offers brief comments on matters arising from the approach, among which we mention the following: elements of $O\left(V_{\mathbb{C}}\right)$ may admit independent generalized Fock implementers, in contrast to the familiar situation for traditional Fock implementers; much of the theory applies not just to $O\left(V_{\mathbb{C}}\right)$ but to those complex-linear transformations of $V_{\mathbb{C}}$ that preserve $(\cdot \mid \cdot)$ but are not necessarily surjective.

For a thorough Clifford algebraic discussion of Fock representations, see $[6]$. Since its appearance in [10], the Shale-Stinespring theorem has received numerous treatments; we mention only [1] and [2]. The Carey-Palmer theorem appears in [4]; [3] and [5] contain related material. [7] presents an alternative approach deserving of further study. See [9] for physical situations in which passage beyond traditional Fock implementation is mandatory. The notion of generalized Fock implementation was investigated for real orthogonal transformations in [8]. The ideas for (1.6) and (1.7) were adapted from [5] and [4] respectively.

\section{§1. Complex Orthogonal Transformations}

Let $V$ be a complex Hilbert space: denote by $J$ its complex structure, by $\langle\cdot \mid \cdot\rangle$ its complex inner product and by $(\cdot \mid \cdot)$ the underlying real inner product; thus, if $x, y \in V$ then

$$
\langle x \mid y\rangle=(x \mid y)+i(J x \mid y)
$$

The complexification $V_{\mathbb{C}}=\mathbb{C} \otimes V=V+i V$ carries a canonical conjugation $\sum$ given by the rule that if $x, y, z \in V$ and $c \in \mathbb{C}$ then $\sum(c \otimes z)=\bar{c} \otimes z$ and 
$\sum(x+i y)=x-i y$. It also carries both a canonical complex inner product $\langle\cdot \mid \cdot\rangle$ given by

$$
\alpha, \beta \in \mathbb{C}, x, y \in V \Rightarrow\langle\alpha \otimes x \mid \beta \otimes y\rangle=\bar{\alpha} \beta(x \mid y)
$$

and a canonical symmetric complex-bilinear form $(\cdot \mid \cdot)$ given by

$$
\alpha, \beta \in \mathbb{C}, x, y \in V \Rightarrow(\alpha \otimes x \mid \beta \otimes y)=\alpha \beta(x \mid y) .
$$

These structures are related by the formula

$$
x, y \in V_{\mathbb{C}} \Rightarrow\langle x \mid y\rangle=(\Sigma x \mid y) .
$$

The complex structure $J: V \rightarrow V$ extends to $J_{\mathbb{C}}: V_{\mathbb{C}} \rightarrow V_{\mathbb{C}}$ by complexlinearity, thereby inducing an eigendecomposition

$$
V_{\mathbb{C}}=V^{+} \oplus V^{-}
$$

in which the eigenspaces

$$
V^{ \pm}=\operatorname{Ker}\left(J_{\mathbb{C}} \mp i I\right)
$$

are both (mutually) perpendicular for $\langle\cdot \mid \cdot\rangle$ and (maximally) isotropic for $(\cdot \mid \cdot)$. Denote the projections of $V_{\mathbb{C}}$ on $V^{+}$and $V^{-}$by $P^{+}=\frac{1}{2}\left(I-i J_{\mathbb{C}}\right)$ and $P^{-}=$ $\frac{1}{2}\left(I+i J_{\mathbb{C}}\right)$ respectively; the bijective restrictions $V \rightarrow V^{+}: v \mapsto v^{+}=P^{+} v$ and $V \rightarrow V^{-}: v \mapsto v^{-}=P^{-} v$ are then complex-linear and antilinear respectively. Note that if $x_{1}, y_{1}, x_{2}, y_{2} \in V$ then

$$
2\left\langle x_{1}^{+}+y_{1}^{-} \mid x_{2}^{+}+y_{2}^{-}\right\rangle=\left\langle x_{1} \mid x_{2}\right\rangle+\left\langle y_{2} \mid y_{1}\right\rangle .
$$

We may express a complex-linear map $G: V_{\mathbb{C}} \rightarrow V_{\mathbb{C}}$ relative to the decomposition $V_{\mathbb{C}}=V^{+} \oplus V^{-}$in block form

$$
G=\left[\begin{array}{ll}
G^{++} & G^{+-} \\
G^{-+} & G^{--}
\end{array}\right]
$$

where $G^{ \pm}: V^{ \pm} \rightarrow V^{ \pm}$and $G^{ \pm \mp}: V^{\mp} \rightarrow V^{ \pm}$. In practice, we prefer to express $G$ in the modified block form

$$
G=\left[\begin{array}{ll}
C^{++}(G) & A^{+-}(G) \\
A^{-+}(G) & C^{--}(G)
\end{array}\right]
$$

defined by the rule that if $v \in V$ then

$$
\begin{aligned}
& G^{ \pm \pm}\left(v^{ \pm}\right)=\left(C^{ \pm \pm}(G) v\right)^{ \pm} \\
& G^{ \pm \mp}\left(v^{\mp}\right)=\left(A^{ \pm \mp}(G) v\right)^{ \pm}
\end{aligned}
$$

whence $C_{G}^{ \pm \pm}=C^{ \pm \pm}(G): V \rightarrow V$ are complex-linear and $A_{G}^{ \pm \mp}=A^{ \pm \mp}(G)$ : $V \rightarrow V$ are antilinear. Of course, this modified block form behaves properly under composition. 
Theorem 1.1. If $H, G: V_{\mathbb{C}} \rightarrow V_{\mathbb{C}}$ are complex-linear then

$$
\begin{aligned}
& C_{H G}^{++}=C_{H}^{++} C_{G}^{++}+A_{H}^{+-} A_{G}^{-+} \\
& C_{H G}^{--}=C_{H}^{--} C_{G}^{--}+A_{H}^{-+} A_{G}^{+-} \\
& A_{H G}^{+-}=A_{H}^{+-} C_{G}^{--}+C_{H}^{++} A_{G}^{+-} \\
& A_{H G}^{-+}=A_{H}^{-+} C_{G}^{++}+C_{H}^{--} A_{G}^{-+} .
\end{aligned}
$$

Proof. Direct calculation from the definitions: for example, if $v \in V$ then

$$
\begin{aligned}
(H G)\left(v^{-}\right)= & H\left(\left(A_{G}^{+-} v\right)^{+}+\left(C_{G}^{--} v\right)^{-}\right) \\
= & \left(C_{H}^{++} A_{G}^{+-} v\right)^{+}+\left(A_{H}^{-+} A_{G}^{+-} v\right)^{-} \\
& +\left(A_{H}^{+-} C_{G}^{--} v\right)^{+}+\left(C_{H}^{--} C_{G}^{--} v\right)^{-}
\end{aligned}
$$

whence projection in $V^{-}$and $V^{+}$yields the central pair of displayed formulae.

Further routine calculation, based on the fact that $\sum=\left[\begin{array}{ll}O & I \\ I & O\end{array}\right]$ in modified block form, establishes that if $G: V_{\mathbb{C}} \rightarrow V_{\mathbb{C}}$ is complex-linear then

$$
\begin{array}{ll}
C^{++}(\Sigma G \Sigma)=C^{--}(G), & A^{+-}(\Sigma G \Sigma)=A^{-+}(G) \\
A^{-+}(\Sigma G \Sigma)=A^{+-}(G), & C^{--}(\Sigma G \Sigma)=C^{++}(G) .
\end{array}
$$

Let us indiscriminately denote by ${ }^{*}$ adjunction relative to the complex inner product $\langle\cdot \mid \cdot\rangle$ on both $V$ and $V_{\mathbb{C}}$.

Theorem 1.2. If $G: V_{\mathbb{C}} \rightarrow V_{\mathbb{C}}$ is complex-linear then

$$
\begin{array}{ll}
C^{++}\left(G^{*}\right)=C^{++}(G)^{*}, & A^{+-}\left(G^{*}\right)=A^{-+}(G)^{*} \\
A^{-+}\left(G^{*}\right)=A^{+-}(G)^{*}, & C^{--}\left(G^{*}\right)=C^{--}(G)^{*} .
\end{array}
$$

Proof. Straightforward calculation from the definitions, based on the fact that if $x_{1}, y_{2}, x_{2}, y_{2} \in V$ then

$$
2\left\langle x_{1}^{+}+y_{1}^{-} \mid x_{2}^{+}+y_{2}^{-}\right\rangle=\left\langle x_{1} \mid x_{2}\right\rangle+\left\langle y_{2} \mid y_{1}\right\rangle .
$$

Let us denote by ${ }^{\top}$ adjunction relative to the complex-bilinear form $(\cdot \mid \cdot)$ on $V_{\mathbb{C}}:$ thus, if $G: V_{\mathbb{C}} \rightarrow V_{\mathbb{C}}$ is complex-linear then

$$
x, y \in V_{\mathbb{C}} \Rightarrow\left(x \mid G^{\top} y\right)=(G x \mid y) .
$$


Theorem 1.3. If $G: V_{\mathbb{C}} \rightarrow V_{\mathbb{C}}$ is complex-linear then

$$
\begin{array}{ll}
C^{++}\left(G^{\top}\right)=C^{--}(G)^{*}, & A^{+-}\left(G^{\top}\right)=A^{+-}(G)^{*} \\
A^{-+}\left(G^{\top}\right)=A^{-+}(G)^{*}, & C^{--}\left(G^{\top}\right)=C^{++}(G)^{*}
\end{array}
$$

Proof. Straightforward calculation from the definitions, based on the fact that if $x_{1}, y_{1}, x_{2}, y_{2} \in V$ then

$$
2\left(x_{1}^{+}+y_{1}^{-} \mid x_{2}^{+}+y_{2}^{-}\right)=\left\langle y_{1} \mid x_{2}\right\rangle+\left\langle y_{2} \mid x_{1}\right\rangle .
$$

Our chief concern is with the complex orthogonal group $O\left(V_{\mathbb{C}}\right)$ comprising all complex-linear automorphisms $G$ of $V_{\mathbb{C}}$ that satisfy $G^{\top}=G^{-1}$ and therefore preserve $(\cdot \mid \cdot)$ in the sense

$$
x, y \in V_{\mathbb{C}} \Rightarrow(G x \mid G y)=(x \mid y) .
$$

We remark that each $G \in O\left(V_{\mathbb{C}}\right)$ is automatically bounded; its $\langle\cdot \mid \cdot\rangle$-adjoint is $G^{*}=\sum G^{-1} \sum$.

As the transformation $G: V_{\mathbb{C}} \rightarrow V_{\mathbb{C}}$ is complex orthogonal precisely when $G^{\top} G=I$ and $G G^{\top}=I$ simultaneously, so the following theorem and subsequent remark are relevant to $O\left(V_{\mathbb{C}}\right)$.

Theorem 1.4. The complex-linear map $G: V_{\mathbb{C}} \rightarrow V_{\mathbb{C}}$ satisfies $G^{\top} G=I$ if and only if it satisfies

$$
\begin{aligned}
& I=\left(C_{G}^{--}\right)^{*} C_{G}^{++}+\left(A_{G}^{+-}\right)^{*} A_{G}^{-+} \\
& I=\left(C_{G}^{++}\right)^{*} C_{G}^{--}+\left(A_{G}^{-+}\right)^{*} A_{G}^{+-} \\
& O=\left(A_{G}^{+-}\right)^{*} C_{G}^{--}+\left(C_{G}^{--}\right)^{*} A_{G}^{+-} \\
& O=\left(A_{G}^{-+}\right)^{*} C_{G}^{++}+\left(C_{G}^{++}\right)^{*} A_{G}^{-+} .
\end{aligned}
$$

Proof. Both directions follow at once upon combining (1.1) and (1.3).

Similarly, the complex-linear map $G: V_{\mathbb{C}} \rightarrow V_{\mathbb{C}}$ satisfies $G G^{\top}=I$ if and only if it satisfies

$$
\begin{aligned}
I & =C_{G}^{--}\left(C_{G}^{++}\right)^{*}+A_{G}^{-+}\left(A_{G}^{+-}\right)^{*} \\
I & =C_{G}^{++}\left(C_{G}^{--}\right)^{*}+A_{G}^{+-}\left(A_{G}^{-+}\right)^{*} \\
O & =A_{G}^{++}\left(C_{G}^{--}\right)^{*}+C_{G}^{--}\left(A_{G}^{-+}\right)^{*} \\
O & =A_{G}^{+-}\left(C_{G}^{++}\right)^{*}+C_{G}^{++}\left(A_{G}^{+-}\right)^{*} .
\end{aligned}
$$


Theorem 1.5. If $G \in O\left(V_{\mathbb{C}}\right)$ then the following diagram is commutative:

$$
\operatorname{Ker} C_{G}^{--} \underset{\left(A_{G}^{-+}\right)^{+}}{\stackrel{A_{G}^{+-}}{\longrightarrow}} \operatorname{Ker}\left(C_{G}^{--}\right)^{*}
$$

Proof. The third of the identities in and after (1.4) imply that $\left(C_{G}^{--}\right)^{*} A_{G}^{+-} \mid \operatorname{Ker} C_{G}^{--}$and $C_{G}^{--}\left(A_{G}^{-+}\right)^{*} \mid \operatorname{Ker}\left(C_{G}^{--}\right)^{*}$ are zero maps while the second of the identities in and after (1.4) imply that $\left(A_{G}^{-+}\right)^{*} A_{G}^{+-} \mid \operatorname{Ker} C_{G}^{--}$and $A_{G}^{+-}\left(A_{G}^{-+}\right)^{*} \mid \operatorname{Ker}\left(C_{G}^{--}\right)^{*}$ are identity maps.

Regarding this result, we make two remarks. First, the formulae of (1.2) enable us to rewrite the commutative diagram in the more symmetric form

$$
\operatorname{Ker} C_{G}^{--} \stackrel{A_{G}^{+-}}{\longleftarrow} \underset{A_{G^{+}}^{+-}}{\longrightarrow} \operatorname{Ker} C_{G^{+}}^{--}
$$

Second, the commutative diagram implies particularly that $\operatorname{Ker} C_{G}^{--}$and $\operatorname{Ker}\left(C_{G}^{--}\right)^{*}$ are equidimensional, so the index of $C_{G}^{--}$is zero when it is defined.

Orthogonal reflections are special elements of $O\left(V_{\mathbb{C}}\right)$. Recall that if $w \in V_{\mathbb{C}}$ satisfies $(w \mid w)=1$ then orthogonal reflection of $V_{\mathbb{C}}$ in the hyperplane perpendicular to $w$ is the element $H \in O\left(V_{\mathbb{C}}\right)$ defined by

$$
v \in V_{\mathbb{C}} \Rightarrow H v=v-2(w \mid v) w .
$$

Theorem 1.6. If $G \in O\left(V_{\mathbb{C}}\right)$ is such that the dimension of $\operatorname{Ker} C_{G}^{--}$is finite and nonzero then there exists a reflection $H \in O\left(V_{\mathbb{C}}\right)$ such that $\operatorname{Ker} C_{H \bar{G}}^{--}$ is a hyperplane in $\operatorname{Ker} C_{G}^{--}$.

Proof. A particular consequence of (1.5) is the existence of $u \in \operatorname{Ker} C_{G}^{--}$ such that $A_{G}^{+-} u \in \operatorname{Ker}\left(C_{G}^{--}\right)^{*}$ is a unit vector. If $H \in O\left(V_{\mathbb{C}}\right)$ is reflection in the hyperplane perpendicular to $A_{G}^{+-} u$ then an elementary calculation reveals that if $v \in V$ then

$$
C_{H G}^{--} v=C_{G}^{--} v-2\left\langle A_{G}^{+-} v \mid A_{G}^{+-} u\right\rangle A_{G}^{+-} u .
$$

As $\operatorname{Ran} C_{G}^{--}$and $\operatorname{Ker}\left(C_{G}^{--}\right)^{*}$ are perpendicular, so it follows that

$$
\operatorname{Ker} C_{H G}^{--}=\left\{v \in \operatorname{Ker} C_{G}^{--}:\left\langle A_{G}^{+-} v \mid A_{G}^{+-} u\right\rangle=0\right\} \text {. }
$$

A further application of (1.5) thus establishes that $\operatorname{Ker} C_{H G}^{--} \subset \operatorname{Ker} C_{G}^{--}$is 
indeed a hyperplane.

Of course, induction on this result proves that if $G \in O\left(V_{\mathbb{C}}\right)$ and $\operatorname{Ker} C_{G}^{--}$ has complex dimension $n$ then there exist $n$ orthogonal reflections whose product $H \in O\left(V_{\mathbb{C}}\right)$ renders $C_{H G}^{--}$injective.

The following result offers an alternative means of eliminating evendimensional kernels.

Theorem 1.7. If $G \in O\left(V_{\mathbb{C}}\right)$ is such that $\operatorname{Ker} C_{G}^{--}$is even-dimensional then there exists an even-rank antilinear endomorphism $h=-h^{*}$ of $V$ such that $H=\left[\begin{array}{ll}I & 0 \\ h & I\end{array}\right] \in O\left(V_{\mathbb{C}}\right)$ renders $C_{H G}^{--}$injective.

Proof. Taking (1.5) into account, the even-dimensionality of $\operatorname{Ker} C_{G}^{--}$ enables us to choose an antilinear automorphism $h=-h^{*}$ of $\operatorname{Ker}\left(C_{G}^{--}\right)^{*}$ such that $h A_{G}^{+-}: \operatorname{Ker} C_{G}^{--} \rightarrow \operatorname{Ker}\left(C_{G}^{--}\right)^{*}$ is an isomorphism. Extend $h$ by zero on the orthocomplement of $\operatorname{Ker}\left(C_{G}^{-}\right)^{*}$ and observe that the modified block operator

$$
H=\left[\begin{array}{ll}
I & O \\
h & I
\end{array}\right]
$$

lies in $O\left(V_{\mathbb{C}}\right)$. We claim that

$$
C_{H G}^{--}=h A_{G}^{+-}+C_{G}^{--}
$$

is injective. To see this, note first that if $v \in V$ then $h A_{G}^{+-} v$ and $C_{G}^{--} v$ are perpendicular: thus, if $C_{H G}^{--} v=0$ then both $C_{G}^{--} v$ and $h A_{G}^{+-} v$ vanish, so the injectivity of $h A_{G}^{+-} \mid \operatorname{Ker} C_{G}^{--}$forces $v=0$.

We remark further that if $\operatorname{Ran} C_{G}^{--}$is closed then $C_{H G}^{--}$is an isomorphism. In fact, decompose $v \in V$ as $v=x+y$ with $x \in \operatorname{Ker}\left(C_{G}^{--}\right)^{*}$ and $y \in \operatorname{Ran} C_{G}^{--}$. As $C_{G}^{--}:\left(\operatorname{Ker} C_{G}^{--}\right)^{\perp} \rightarrow \operatorname{Ran} C_{G}^{--}$is surjective, so there exists $y_{0} \in\left(\operatorname{Ker} C_{G}^{--}\right)^{\perp}$ such that $C_{G}^{--} y_{0}=y$. As $h A_{G}^{+-}: \operatorname{Ker} C_{G}^{--} \rightarrow \operatorname{Ker}\left(C_{G}^{--}\right)^{*}$ is surjective, so there exists $x_{0} \in \operatorname{Ker} C_{G}^{--}$such that $h A_{G}^{+-} x_{0}=x-h A_{G}^{+-} y_{0}$. Assembling the pieces, $C_{H G}^{--}\left(x_{0}+y_{0}\right)=x+y=v$.

Finally, we consider briefly the real orthogonal group $O(V)$ comprising all real-linear automorphisms $g$ of $V$ such that $g^{*}=g^{-1}$. Of course, complexification embeds $O(V)$ in $O\left(V_{\mathbb{C}}\right)$.

Theorem 1.8. If $g \in O(V)$ then $G=g_{\mathbb{C}}$ lies in $O\left(V_{\mathbb{C}}\right)$ and satisfies $G \sum=\sum G ;$ conversely, each element of $O\left(V_{\mathbb{C}}\right)$ that commutes with $\sum$ arises in this way. 
Proof. Elementary: the forward implication holds essentially by the complex-bilinearity of $(\cdot \mid \cdot)$ on $V_{\mathbb{C}}$; the reverse implication holds because complexlinear endomorphisms of $V_{\mathbb{C}}$ commuting with $\sum$ stabilize $V$.

By direct calculation, if the real-linear endomorphism $g$ of $V$ is extended to $G=g_{\mathbb{C}}$ by complexification, then $C_{G}^{++}$and $C_{G}^{--}$coincide with the complexlinear part $C_{g}=\frac{1}{2}(g-J g J)$ while $A_{G}^{+-}$and $A_{G}^{-+}$coincide with the antilinear part $A_{g}=\frac{1}{2}(g+J g J)$.

\section{§2. Generalized Fock Implementation}

The carrier space for the Fock representation of $V$ develops from its exterior algebra $\wedge V$. Recall that $\wedge V$ is graded by degree:

$$
\wedge V=\bigoplus_{n \in \mathbb{N}} \wedge^{n} V
$$

where $\wedge^{\circ} V=\mathbb{C}$ and where if $n>0$ then $\wedge^{n} V$ is spanned by the decomposables $v_{1} \wedge \ldots \wedge v_{n}$ for $v_{1}, \ldots, v_{n} \in V$. Recall also that $\wedge V$ has a standard complex inner product $\langle\cdot \mid \cdot\rangle$ for which the homogeneous summands are perpendicular, the Fock vacuum $1 \in \wedge^{\circ} V$ is a unit vector and if $x_{1}, \ldots, x_{n}, y_{1}, \ldots, y_{n} \in V$ then

$$
\left\langle x_{1} \wedge \ldots \wedge x_{n} \mid y_{1} \wedge \ldots \wedge y_{n}\right\rangle=\operatorname{Det}\left[\left\langle x_{a} \mid y_{b}\right\rangle\right]
$$

By definition, fermionic Fock space $\wedge[V]$ is the complex Hilbert space completion of $\wedge V$ relative to this inner product.

It is illuminating to consider alongside the exterior algebra $\wedge V$ its full antidual $\wedge V^{\prime}$ comprising all antilinear functionals $\wedge V \rightarrow \mathbb{C}$. The full antidual is naturally an associative algebra: the diagonal map $V \rightarrow V \oplus V$ induces an algebra homomorphism $\wedge V \rightarrow \wedge(V \oplus V)$ which when followed by the canonical isomorphism $\wedge(V \oplus V) \rightarrow \wedge V \otimes \wedge V$ yields a (coassociative) coproduct $\Delta$ : $\wedge V \rightarrow \wedge V \oplus \wedge V$; this determines a canonical (associative) product in $\wedge V^{\prime}$ according to which if $\Phi, \Psi \in \wedge V^{\prime}$ and $\theta \in \wedge V$ then

$$
[\Phi \Psi](\theta)=[\Phi \otimes \Psi](\Delta \theta) .
$$

The full antidual naturally includes the exterior algebra as a subalgebra: indeed, an algebra embedding is induced by the standard inner product, thus

$$
\wedge V \rightarrow \wedge V^{\prime \prime}: \phi \mapsto\langle\cdot \mid \phi\rangle
$$


In fact, $\wedge V^{\prime}$ includes a canonical version of $\wedge[V]$ : namely, the space comprising all bounded antilinear functionals on $\wedge V$; thus $\wedge V \subset \wedge[V] \subset \wedge V^{\prime}$ is a Gelfand triple.

When $v \in V$ we define the creator $c(v): \wedge V \rightarrow \wedge V$ to be the operator of left multiplication by $v$ so that

$$
\phi \in \wedge V \Rightarrow c(v) \phi=v \wedge \phi
$$

and define the annihilator $a(v): \wedge V \rightarrow \wedge V$ to be the linear antiderivation annihilating the Fock vacuum and satisfying

$$
w \in V \Rightarrow a(v) w=\langle v \mid w\rangle
$$

whence in particular if $v_{0}, \ldots, v_{n} \in V$ then

$$
a(v)\left(v_{0} \wedge \ldots \wedge v_{n}\right)=\sum_{k=0}^{n}(-1)^{k}\left\langle v \mid v_{k}\right\rangle v_{0} \wedge \ldots \wedge \hat{v}_{k} \wedge \ldots \wedge v_{n}
$$

where $\hat{\imath}$ signifies omission as usual. A straightforward determinantal calculation reveals that these operators are mutually adjoint in the sense that if $v \in V$ then

$$
\phi, \psi \in \wedge V \Rightarrow\langle a(v) \phi \mid \psi\rangle=\langle\phi \mid c(v) \psi\rangle
$$

Accordingly, when $v \in V$ we extend $c(v)$ and $a(v)$ to the antidual by declaring that if $\Phi \in \wedge V^{\prime}$ and $\psi \in \wedge V$ then

$$
\begin{aligned}
& {[c(v) \Phi](\psi)=\Phi(a(v) \psi)} \\
& {[a(v) \Phi](\psi)=\Phi(c(v) \psi) .}
\end{aligned}
$$

These extended operators are evidently continuous when $\wedge V^{\prime}$ is equipped with the weak (evaluative) topology.

Recall that if $X$ and $Y$ are linear operators on a vector space then their anticommutator is defined by

$$
\{X, Y\}=X Y+Y X .
$$

Theorem 2.1. Creators and annihilators satisfy the canonical anticommutation relations on $\wedge V$ and $\wedge V^{\prime}:$ if $x, y \in V$ then

$$
\begin{aligned}
& \{a(x), a(y)\}=0 \\
& \{a(x), c(y)\}=\langle x \mid y\rangle I \\
& \{c(x), c(y)\}=0 .
\end{aligned}
$$


Proof. Validity on $\wedge V^{\prime}$ follows by antiduality from validity on $\wedge V$. Regarding validity on $\wedge V$ itself, the last relation holds by anticommutativity of exterior product and the first relation holds by adjunction, while the central relation holds because if $\phi \in \wedge V$ then

$$
\begin{aligned}
a(x) c(y) \phi & =a(x)(y \wedge \phi) \\
& =[a(x) y] \phi-y \wedge[a(x) \phi] \\
& =\langle x \mid y\rangle \phi-c(y) a(x) \phi .
\end{aligned}
$$

As a particular consequence of the canonical anticommutation relations and the mutually adjoint nature of creators and annihilators, if $v \in V$ and $\phi \in \wedge V$ then

$$
\|c(v) \phi\|^{2}+\|a(v) \phi\|^{2}=\|v\|^{2}\|\phi\|^{2}
$$

whence $c(v)$ and $a(v)$ are bounded linear operators on $\wedge V$. It is not difficult to verify that the continuous extensions of $c(v)$ and $a(v)$ from $\wedge V$ to $\wedge[V]$ coincide respectively with the restrictions of $c(v)$ and $a(v)$ from $\wedge V^{\prime}$ to $\wedge[V]$.

After these preliminaries, we may introduce the Fock representation itself. Traditionally, this associates to each $v \in V$ the map

$$
\pi(v)=c(v)+a(v): \wedge[V] \rightarrow \wedge[V]
$$

which is a selfadjoint bounded linear operator whose square is scalar multiplication by $\|v\|^{2}$. Though it is not important for our account, it follows that $\pi$ extends to define a representation of the $C^{*}$ Clifford algebra of $V$ on $\wedge[V]$. For our purposes, it is important that $\pi$ extends to $V_{\mathbb{C}}$ by complex-linearity: thus, if $x, y \in V$ then

$$
\begin{aligned}
\pi(x+i y) & =\pi(x)+i \pi(y) \\
\pi\left(x^{+}+y^{-}\right) & =c(x)+a(y) .
\end{aligned}
$$

Passing beyond tradition, when $v \in V$ we may regard $\pi(v)=c(v)+a(v)$ as a linear endomorphism not only of fermionic Fock space $\wedge[V]$ but also of the exterior algebra and its full antidual. Thus we obtain $\pi$ as a representation of $V$ on the triple $\wedge V \subset \wedge[V] \subset \wedge V^{\prime}$ which we call the generalized Fock representation. This representation also extends to $V_{\mathbb{C}}$ by complex-linearity: note that if $v \in V_{\mathbb{C}}$ then

$$
\Phi \in \wedge V^{\prime}, \psi \in \wedge V \Rightarrow[\pi(v) \Phi](\psi)=\Phi(\pi(\Sigma v) \psi)
$$


and that if $x, y \in V_{\mathbb{C}}$ then

$$
\{\pi(x), \pi(y)\}=2(x \mid y) I .
$$

Implementability in the Fock representation was initially considered for real orthogonal transformations. The celebrated Shale-Stinespring theorem [10] establishes that if $g \in O(V)$ then there exists a unitary operator $U$ on $\wedge[V]$ such that

$$
v \in V \Rightarrow U \pi(v)=\pi(g v) U
$$

precisely when the antilinear part $A_{g}=\frac{1}{2}(g+J g J)$ is of Hilbert-Schmidt class. Fock implementability of complex orthogonal transformations is decidedly less straight-forward. In [4] Carey and Palmer construct a $\pi$-invariant dense complex subspace $\mathcal{D} \subset \wedge\left[V^{r}\right]$ and to each $G \in O\left(V_{\mathbb{C}}\right)$ for which not only $A_{G}^{ \pm \mp}$ are Hilbert-Schmidt but also $C_{G}^{ \pm \pm}-I$ are trace-class they associate a linear automorphism $U$ of $\mathcal{D}$ such that

$$
v \in V_{\mathbb{C}} \Rightarrow U \pi(v)=\pi(G v) U
$$

Here we shall proceed in an essentially algebraic manner, replacing fermionic Fock space $\wedge[V]$ by the exterior algebra $\wedge V$ and its full antidual $\wedge V^{\prime}$. On the one hand, we shall thereby develop the Shale-Stinespring and Carey-Palmer theorems as far as may be reasonably expected. On the other hand, we shall have to sacrifice some of their representation-theoretic aspects.

By way of preparation, it is technically convenient to introduce transformed creators and annihilators. Thus, let $G \in O\left(V_{\mathbb{C}}\right)$ and let $v \in V$. We define

$$
\begin{aligned}
& c_{G}(v)=c\left(C_{G}^{++} v\right)+a\left(A_{G}^{-+} v\right) \\
& a_{G}(v)=c\left(A_{G}^{+-} v\right)+a\left(C_{G}^{--} v\right)
\end{aligned}
$$

as linear operators on $\wedge V$. These are not generally mutual adjoints: rather, elementary calculation involving the formulae after (1.1) reveals that if $\phi, \psi, \in$ $\wedge V$ then

$$
\left\langle a_{G}(v) \phi \mid \psi\right\rangle=\left\langle\phi \mid c_{\sum G \sum}(v) \psi\right\rangle .
$$

Accordingly, we extend $c_{G}(v)$ and $a_{G}(v)$ to linear operators on $\wedge V^{\prime}$ by declaring that if $\Phi \in \wedge V^{\prime}$ and $\psi \in \wedge V$ then

$$
\begin{aligned}
& {\left[c_{G}(v) \Phi\right](\psi)=\Phi\left(a_{\sum G \sum}(v) \psi\right)} \\
& {\left[a_{G}(v) \Phi\right](\psi)=\Phi\left(c_{\sum G \sum}(v) \psi\right)}
\end{aligned}
$$


These transformed creators and annihilators continue to satisfy the canonical anticommutation relations on both $\wedge V$ and $\wedge V^{\prime}$.

Theorem 2.2. If $G \in O\left(V_{\mathbb{C}}\right)$ and $x, y \in V$ then

$$
\begin{aligned}
& \left\{a_{G}(x), a_{G}(y)\right\}=0 \\
& \left\{a_{G}(x), c_{G}(y)\right\}=\langle x \mid y\rangle I \\
& \left\{c_{G}(x), c_{G}(y)\right\}=0 .
\end{aligned}
$$

Proof. As an illustration, take the central identity: from the original canonical anticommutation relations (2.1) and the first identity of (1.4) it follows that

$$
\begin{aligned}
\left\{a_{G}(x), c_{G}(y)\right\} & =\left\{c\left(A_{G}^{+-} x\right)+a\left(C_{G}^{--} x\right), c\left(C_{G}^{++} y\right)+a\left(A_{G}^{-+} y\right)\right\} \\
& =\left[\left\langle C_{G}^{--} x \mid C_{G}^{++} y\right\rangle+\left\langle A_{G}^{-+} y \mid A_{G}^{+-} x\right\rangle\right] I \\
& =\left\langle x \mid\left[\left(C_{G}^{--}\right)^{*} C_{G}^{++}+\left(A_{G}^{+-}\right)^{*} A_{G}^{-+}\right] y\right\rangle I \\
& =\langle x \mid y\rangle I .
\end{aligned}
$$

The relevance of transformed creators and annihilators to Fock implementation is evident from the next result.

Theorem 2.3. If $G \in O\left(V_{\mathbb{C}}\right)$ and $v \in V$ then

$$
\pi(G v)=c_{G}(v)+a_{G}(v) .
$$

Proof. Recall that if $x, y \in V$ then $\pi\left(x^{+}\right)=c(x)$ and $\pi\left(y^{-}\right)=a(y)$. Accordingly, from

$$
\begin{aligned}
G v & =G\left(v^{+}\right)+G\left(v^{-}\right) \\
& =\left(C_{G}^{++} v\right)^{+}+\left(A_{G}^{-+} v\right)^{-}+\left(A_{G}^{+-} v\right)^{+}+\left(C_{G}^{--} v\right)^{-}
\end{aligned}
$$

it follows that

$$
\begin{aligned}
\pi(G v) & =\pi\left[\left(C_{G}^{++} v+A_{G}^{+-} v\right)^{+}\right]+\pi\left[\left(A_{G}^{-+} v+C_{G}^{--} v\right)^{-}\right] \\
& =c\left(C_{G}^{++} v+A_{G}^{+-} v\right)+a\left(A_{G}^{-+} v+C_{G}^{--} v\right) \\
& =c_{G}(v)+a_{G}(v) .
\end{aligned}
$$


Now, by a generalized Fock implementer for $G \in O\left(V_{\mathbb{C}}\right)$ we shall mean a (nonzero) complex-linear map $U: \wedge V \rightarrow \wedge V^{\prime}$ such that

$$
v \in V_{\mathbb{C}} \Rightarrow U \pi(v)=\pi(G v) U
$$

or equivalently

$$
v \in V \Rightarrow\left\{\begin{array}{l}
U c(v)=c_{G}(v) U \\
U a(v)=a_{G}(v) U
\end{array}\right.
$$

and by a generalized Fock vacuum for $G \in O\left(V_{\mathbb{C}}\right)$ we shall mean a (nonzero) vector $\Phi \in \wedge V^{\prime}$ such that

$$
v \in V \Rightarrow a_{G}(v) \Phi=0
$$

Theorem 2.4. Let $G \in O\left(V_{\mathbb{C}}\right)$ : the rule $\Phi=U 1$ establishes a bijection between its generalized Fock vacua $\Phi \in \wedge V^{\prime}$ and its generalized Fock implementers $U: \wedge V \rightarrow \wedge V^{\prime}$; this bijection has the property that if $v_{1}, \ldots, v_{n} \in V$ then

$$
U\left(v_{1} \wedge \ldots \wedge v_{n}\right)=c_{G}\left(v_{1}\right) \ldots c_{G}\left(v_{n}\right) \Phi
$$

Proof. In the one direction, if $U$ is a generalized Fock implementer and if $v \in V$ then $a_{G}(v) U 1=U a(v) 1=0$ so that $U 1$ is a generalized Fock vacuum. In the other direction, if $\Phi$ is a generalized Fock vacuum then the canonical anticommutation relations of (2.2) guarantee that $U 1:=\Phi$ and the indicated rule

$$
v_{1}, \ldots, v_{n} \in V \Rightarrow U\left(v_{1} \wedge \ldots \wedge v_{n}\right)=c_{G}\left(v_{1}\right) \ldots c_{G}\left(v_{n}\right) \Phi
$$

together well-define a generalized Fock implementer. Of course, the correspondence $\Phi \leftrightarrow U$ is bijective.

It is an elementary fact that if $\Phi \in \wedge V^{\prime}$ and if each vector $w$ in the infinite-dimensional subspace $W \subset V$ satisfies $c(w) \Phi=0$ then $\Phi=0$ : indeed, if $v_{1}, \ldots, v_{n} \in V$ then choosing a unit vector $w \in W$ perpendicular to each yields not only

$$
\begin{aligned}
\Phi\left(v_{1} \wedge \ldots \wedge v_{n}\right) & =\Phi\left(a(w)\left(w \wedge v_{1} \wedge \ldots \wedge v_{n}\right)\right) \\
& =[c(w) \Phi]\left(w \wedge v_{1} \wedge \ldots \wedge v_{n}\right)=0
\end{aligned}
$$


but also

$$
\Phi(1)=\Phi(a(w) w)=[c(w) \Phi](w)=0 .
$$

This elementary fact leads at once to a necessary condition for the existence of generalized Fock implementers.

Theorem 2.5. If $G \in O\left(V_{\mathbb{C}}\right)$ admits a generalized Fock implementer then $\operatorname{Ker} C^{--}(G)$ is finite-dimensional.

Proof. Let the (nonzero) vector $\Phi \in \wedge V^{\prime}$ be a generalized Fock vacuum for $G$ : if $v \in \operatorname{Ker} C^{--}(G)$ then

$$
c\left(A^{+-}(G) v\right) \Phi=a_{G}(v) \Phi=0
$$

whence the fact recorded prior to the theorem implies that $A^{+-}(G)\left[\operatorname{Ker} C^{--}(G)\right]$ is finite-dimensional; an application of (1.5) concludes the proof.

As a matter of fact, this condition for the existence of generalized Fock implementers is not only necessary but also sufficient; to see that this is so, we proceed by stages.

At this point, it is convenient to discuss briefly Gaussians in a generalized sense. Thus, to each antilinear map $Z: V \rightarrow V^{\prime}$ that is antiskew in the sense

$$
x, y \in V \Rightarrow Z x(y)+Z y(x)=0
$$

let us associate the quadratic $\zeta \in \wedge V^{\prime}$ vanishing except in second degree and satisfying

$$
x, y \in V \Rightarrow \zeta(x \wedge y)=Z x(y)
$$

The (generalized) Gaussian

$$
e^{Z}=\sum_{n \in \mathbb{N}} \frac{1}{n !} \zeta^{n} \in \wedge V^{\prime}
$$

converges weakly since each element of $\wedge V$ vanishes in sufficiently high degree. Note that if $v \in V$ then by induction

$$
n \in \mathbb{N} \Rightarrow a(v) \zeta^{n}=n(Z v) \zeta^{n-1}
$$

whence by summation

$$
a(v) e^{Z}=(Z v) e^{Z} .
$$


With this understanding of Gaussians, we may be very explicit about generalized Fock implementers for a significant class of complex orthogonal transformations.

Theorem 2.6. Each $G \in O\left(V_{\mathbb{C}}\right)$ for which $C^{--}(G)$ is injective admits a generalized Fock implementer whose corresponding generalized Fock vacuum is a Gaussian.

Proof. Choose a subspace $W^{\top} \subset V$ complementary to $\operatorname{Ran} C_{G}^{--}$in the algebraic sense. Define the antilinear map $Z: V \rightarrow V^{\prime}$ by the rule that if $w, w_{0} \in W$ and $v, v_{0} \in V$ then

$$
Z\left(w_{0}+C_{G}^{--} v_{0}\right)\left(w+C_{G}^{--} v\right)=\left\langle C_{G}^{--} v_{0} \mid A_{G}^{+-} v\right\rangle+\left\langle w_{0} \mid A_{G}^{+-} v\right\rangle-\left\langle w \mid A_{G}^{+-} v_{0}\right\rangle .
$$

The map $Z$ is antiskew, for the third identity of (1.4) shows that if $x, y \in V$ then

$$
\left\langle C_{G}^{--} x \mid A_{G}^{+-} y\right\rangle+\left\langle C_{G}^{--} y \mid A_{G}^{+-} x\right\rangle=\left\langle x_{\mid} \mid\left[\left(C_{G}^{--}\right)^{*} A_{G}^{+-}+\left(A_{G}^{+-}\right)^{*} C_{G}^{--}\right] y\right\rangle=0 .
$$

The map $Z$ also satisfies

$$
A_{G}^{+-}+Z C_{G}^{--}=0
$$

for if $w \in W^{*}$ and $v, v_{0} \in V$ then

$$
\begin{aligned}
Z\left(C_{G}^{--} v_{0}\right)\left(w+C_{G}^{--} v\right) & =\left\langle C_{G}^{--} v_{0} \mid A_{G}^{+-} v\right\rangle-\left\langle w \mid A_{G}^{+-} v_{0}\right\rangle \\
& =-\left\langle w+C_{G}^{--} v \mid A_{G}^{+-} v_{0}\right\rangle .
\end{aligned}
$$

Thus, if $\imath^{\prime} \in V$ then

$$
\begin{aligned}
a_{G}(v) e^{Z} & =\left[c\left(A_{G}^{+-} v\right)+a\left(C_{G}^{--} v\right)\right] e^{Z} \\
& =\left(A_{G}^{+-} v+Z C_{G}^{--} v\right) e^{Z}=0
\end{aligned}
$$

and so the Gaussian $e^{Z}$ is a generalized Fock vacuum for $G$.

Fock implementers for complex orthogonal reflections may be described explicitly using the parity operator $\Gamma: \wedge V \rightarrow \wedge V$ which multiplies elements of degree $n \in \mathbb{N}$ by $(-1)^{n}$.

Theorem 2.7. If $w \in V_{\mathbb{C}}$ is a unit vector then $\pi(w) \Gamma: \wedge V \rightarrow \wedge V$ is a Fock implementer for orthogonal reflection of $V_{\mathbb{C}}$ in the hyperplane perpendicular to $w$. 
Proof. Let $H \in O\left(V_{\mathbb{C}}\right)$ be orthogonal reflection of $V_{\mathbb{C}}$ in the hyperplane perpendicular to $w$. As $\pi(w)^{2}=I$ so if $v \in V_{\mathbb{C}}$ then from

$$
\pi(w) \pi(v)+\pi(v) \pi(w)=2(w \mid v) I
$$

it follows directly that

$$
\pi(w) \pi(v)=\pi(2(w \mid v) w-v) \pi(w)=-\pi(H v) \pi(w)
$$

whence $\{\Gamma, \pi(v)\}=0$ implies

$$
\pi(w) \Gamma \pi(v)=-\pi(w) \pi(v) \Gamma=\pi(H v) \pi(w) \Gamma .
$$

We remark that here, the implementing operator on $\wedge V$ extends to an implementing operator on $\wedge V^{\prime}$ by antiduality: if $w \in V_{\mathbb{C}}$ then

$$
\Phi \in \wedge V^{\prime}, \psi \in \wedge V \Rightarrow[\pi(w) \Phi](\psi)=\Phi(\pi(\Sigma w) \psi)
$$

while $\Gamma: \wedge V \rightarrow \wedge V$ is self-adjoint so

$$
\Phi \in \wedge V^{\prime}, \psi \in \wedge V \Rightarrow[\Gamma \Phi](\psi)=\Phi(\Gamma \psi) .
$$

We remark further that by induction, each $H \in O\left(V_{\mathbb{C}}\right)$ that is a finite product of orthogonal reflections admits a Fock implementer $U: \wedge V \rightarrow \wedge V$ which extends to a Fock implementer $U: \wedge V^{\prime} \rightarrow \wedge V^{\prime}$ by antiduality, thus

$$
\Phi \in \wedge V^{\prime}, \psi \in \wedge V \Rightarrow[U \Phi](\psi)=\Phi\left(U^{*} \psi\right) .
$$

Finally, we are able to prove the following converse of (2.5).

Theorem 2.8. Each $G \in O\left(V_{\mathbb{C}}\right)$ for which $\operatorname{Ker} C^{--}(G)$ is finite-dimensional admits a generalızed Fock implementer.

Proof. This may be formalized as an induction on the complex dimension of $\operatorname{Ker} C^{--}(G)$. The base step is provided by (2.6) of course. For the inductive step, note first that (1.6) furnishes a complex orthogonal reflection $H \in O\left(V_{\mathbb{C}}\right)$ such that $\operatorname{Ker} C^{--}(H G)$ is a hyperplane in $\operatorname{Ker} C^{--}(G)$ : now, $H$ admits a Fock implementer $U_{H}: \wedge V^{\prime} \rightarrow \wedge V^{\prime}$ by the first remark after (2.7) and $H G$ admits a generalized Fock implementer $U_{H G}: \wedge V \rightarrow \wedge V^{\prime}$ by the inductive hypothesis; as is readily verified, the composite $U_{H} \circ U_{H G}: \wedge V \rightarrow \wedge V^{\prime}$ is a generalized Fock implementer for $H \circ H G=G$. 


\section{§3. Remarks}

Here we offer a number of brief comments on issues arising from the body of the paper.

Recall that as regards traditional Fock implementation in (say) the ShaleStinespring context, the unitary implementers (equivalently, the displaced vacua) are unique up to scalar multiples. By way of contrast, it is interesting (perhaps surprising) to observe that the situation regarding generalized Fock implementation can be radically different. To take a simple example, let $G \in O\left(V_{\mathbb{C}}\right)$ be such that $C^{--}(G)$ is injective but not surjective. Let $\operatorname{Ran} C^{--}(G)$ have $W \subset V$ as an algebraic complement and let $z: W \rightarrow W^{\prime}$ be any antiskew map. A glance at the proof of (2.6) reveals that $G$ admits as generalized Fock vacuum the (Gaussian) exponential of the antiskew map $Z+z: V \rightarrow V^{\prime}$ defined by the rule that if $w, w_{0} \in W$ and $v, v_{0} \in V$ then

$$
\begin{gathered}
(Z+z)\left(w_{0}+C_{G}^{--} v_{0}\right)\left(w+C_{G}^{--} v\right)= \\
z\left(w_{0}\right)(w)+\left\langle C_{G}^{--} v_{0} \mid A_{G}^{+-} v\right\rangle+\left\langle w_{0} \mid A_{G}^{+-} v\right\rangle-\left\langle w \mid A_{G}^{+-} v_{0}\right\rangle .
\end{gathered}
$$

In fact, the existence of independent displaced vacua is not limited to complex orthogonal transformations: it already occurs for real orthogonal transformations. Let $V$ be infinite-dimensional, let $K \in O(V)$ be a quaternionic (that is, antilinear complex) structure and let

$$
V=\bigoplus_{n \in \mathbb{N}} V_{n}
$$

be an orthogonal decomposition into $K$-invariant complex subspaces. Choose a real sequence $\left(\theta_{n}: n \in \mathbb{N}\right)$ such that the sequence $\left(\cos \theta_{n}: n \in \mathbb{N}\right)$ is (say) strictly positive and converges to zero. It is readily verified that if

$$
g=\bigoplus_{n \in N}\left(e^{\theta_{n} K}\right) \in O(V)
$$

then $C_{g}=\oplus_{n \in N}\left(\cos \theta_{n}\right) I$ is injective but has zero in its spectrum.

Let us extend the notion of adjunction, declaring that the adjoint of the complex-linear map $U: \wedge V \rightarrow \wedge V^{\prime}$ is the complex-linear map $U^{*}: \wedge V \rightarrow \wedge V^{\prime}$ defined by

$$
\phi, \psi \in \wedge V \Rightarrow\left[U^{*} \phi\right](\psi)=\overline{[U \psi](\phi)}
$$

With this declaration, if $G \in O\left(V_{\mathbb{C}}\right)$ has $U: \wedge V \rightarrow \wedge V^{\prime}$ as generalized Fock implementer then $G^{*}=\sum G^{-1} \sum$ has $U^{*}$ as generalized Fock implementer. To 
see this, note first that if $v \in V$ then (1.3) and the remark after (1.4) imply that

$$
\begin{gathered}
c_{G}\left(C_{G^{-1}}^{++} v\right)+a_{G}\left(A_{G^{-1}}^{-+} v\right)= \\
c\left(C_{G}^{++} C_{G^{-1}}^{++} v\right)+a\left(A_{G}^{-+} C_{G^{-1}}^{++} v\right)+c\left(A_{G}^{+-} A_{G^{-1}}^{-+} v\right)+a\left(C_{G}^{--} A_{G^{-1}}^{-+} v\right) \\
=c(v)
\end{gathered}
$$

so that

$$
\begin{aligned}
U c_{G^{-1}}(v) & =U\left[c\left(C_{G^{-1}}^{++} v\right)+a\left(A_{G^{-1}}^{-+} v\right)\right] \\
& =\left[c_{G}\left(C_{G^{-1}}^{++} v\right)+a_{G}\left(A_{G^{-1}}^{-+} v\right)\right] U \\
& =c(v) U
\end{aligned}
$$

whence if also $\phi, \psi \in \wedge V$ then

$$
\begin{aligned}
{\left[U^{*} a(v) \phi\right](\psi) } & =\overline{[U \psi](a(v) \phi)} \\
& =\overline{[c(v) U \psi](\phi)} \\
& =\overline{\left[U c_{G^{-1}}(v) \psi\right](\phi)} \\
& =\left[U^{*} \phi\right]\left(c_{G^{-1}}(v) \psi\right) \\
& =\left[a_{\Sigma^{G^{-1}} \Sigma}(v) U^{*} \phi\right](\psi) .
\end{aligned}
$$

Accordingly, if $v \in V$ then

$$
U^{*} a(v)=a_{G^{*}}(v) U^{*}
$$

and similarly

$$
U^{*} c(v)=c_{G^{*}}(v) U^{*}
$$

It is natural to ask why $C^{--}$but not $C^{++}$should be revelant to generalized Fock implementation. One answer to this question involves the anti-Fock representation. For this, let $V$ have conujugate space $\bar{V}$ : the same real inner product space but with reversed complex structure and complex inner product; the corresponding eigendecomposition $\bar{V}_{\mathbb{C}}=\bar{V}^{+} \oplus \bar{V}^{-}$has $\bar{V}^{+}=V^{-}$and $\bar{V}^{-}=V^{+}$. The anti-Fock representation $\bar{\pi}$ of $V$ is by definition the Fock representation of $\bar{V}$ : thus, if $v \in V$ then $\bar{\pi}(v)=\bar{c}(v)+\bar{a}(v)$ where (initially) $\bar{c}(v)$ is left multiplication by $v$ on $\wedge \bar{V}$ and $\bar{a}(v)$ is the linear antiderivation of $\wedge \bar{V}$ annihilating 1 and satisfying $\bar{a}(v) \mid \bar{V}=\langle\cdot \mid v\rangle$. Now, each $G \in O\left(\bar{V}_{\mathbb{C}}\right)$ may be regarded as $\bar{G} \in O\left(\bar{V}_{\mathbb{C}}\right)$ with $C^{ \pm \pm}(\bar{G})=C^{\mp \mp}(G)$ and $A^{ \pm \mp}(\bar{G})=A^{\mp \pm}(G)$. Accordingly, it follows from (2.5) and (2.8) that $G$ admits generalized anti-Fock implementers 
precisely when $\operatorname{Ker} C^{++}(G)$ is finite-dimensional. Another answer is based on the observation that if $G \in O\left(V_{\mathbb{C}}\right)$ then $C^{--}\left(G^{-1}\right)=C^{--}\left(G^{\top}\right)=C^{++}(G)^{*}$ by (1.3) whence $\operatorname{Ker} C^{--}\left(G^{-1}\right)$ and $\operatorname{Ker} C^{++}(G)$ are equidimensional by (1.5). Accordingly, it follows that if $G \in O\left(V_{\mathbb{C}}\right)$ then $G^{-1}$ admits generalized Fock implementers precisely when $\operatorname{Ker} C^{++}(G)$ is finite-dimensional.

In both the Shale-Stinespring and Carey-Palmer contexts, the Fock implemented orthogonal transformations constitute a group. By way of contrast, the orthogonal transformations admitting generalized Fock implementers do not constititute a group in infinite dimensions. Thus, it is possible for $G \in O\left(V_{\mathbb{C}}\right)$ to admit generalized Fock implementers while $G^{-1}$ does not: we need only exhibit a $G$ for which $\operatorname{Ker} C^{--}(G)$ is finite-dimensional while $\operatorname{Ker} C^{++}(G)$ is not; for example, (1.4) and the subsequent remark show that such arises upon choosing a quaternionic structure $K \in O(V)$ and setting

$$
G=\left[\begin{array}{ll}
O & K \\
K & I
\end{array}\right] \in O\left(V_{\mathbb{C}}\right) .
$$

Observe that this phenomenon cannot occur for real orthogonal transformations, for if $g \in O(V)$ then $C^{++}\left(g_{\mathbb{C}}\right)=C^{--}\left(g_{\mathbb{C}}\right)=C_{g}$. Also, it is possible for elements of $O\left(V_{\mathbb{C}}\right)$ to admit generalized Fock implementers while their product does not. In fact, this phenomenon even occurs for real orthogonal transformations: if $K \in O(V)$ is a quaternionic structure then $g=\frac{1}{\sqrt{2}}\left(I+K^{r}\right) \in O(V)$ admits generalized Fock implementers $\left(C_{g}=\frac{1}{\sqrt{2}} I\right.$ is injective) while $g^{2}$ does not $\left(C_{g^{2}}\right.$ is zero).

It is worth pointing out that many of the results in the paper extend beyond the complex orthogonal group. For instance, let $G: V_{\mathbb{C}} \rightarrow V_{\mathbb{C}}$ be a complex-linear map that preserves the symmetric complex-bilinear form $(\cdot \mid \cdot)$ in the sense

$$
x, y \in V_{\mathbb{C}} \Rightarrow(G x \mid G y)=(x \mid y)
$$

but do not suppose that $G$ is surjective: thus, assume $G^{\top} G=I$ but do not insist upon $G G^{\top}=I$, so (1.4) applies but the subsequent remark need not. As before, define a generalized Fock vacuum for $G$ to be a nonzero vector $\Phi \in V^{\prime}$ such that

$$
v \in V \Rightarrow a_{G}(v) \Phi=0 .
$$

A glance at the proof of (2.5) reveals that it continues to apply in this context: if $G$ admits generalized Fock vacua then $\operatorname{Ker} C^{--}(G)$ is finite-dimensional. Conversely, if $\operatorname{Ker} C^{--}(G)$ is finite-dimensional then $G$ admits generalized Fock 
vacua essentially as in (2.8); the proofs of (1.6) and (2.6) carry over without substantial change. .

\section{References}

[1] Araki, H., Bogoliubov automorphisms and Fock representations of canonical anticommutation relations, Contemp. Math., 62 (1987), 23-141.

[2] Berezin, F. A., The method of second quantızatıon, Academic Press, 1966.

[3] Carey, A., Spin groups and Clifford algebras, L.M.S. Lecture Notes, 136 (1988), 77101.

[4] Carey, A. and Palmer, J., Infinite Complex Spin Groups, J. Funct. Anal., 83 (1989), $1-43$.

[5] Palmer, J., A Grassmann calculus for infinite spin groups, J. Math. Phys., 29 (1988), 1283-1299.

[6] Plymen, R. J. and Robinson, P. L., Spmors in Hulbert space, Cambridge University Press, 1994

[7] Pressley, A. and Segal, G., Loop Groups, Oxford University Press, 1986.

[8] Robinson, P. L., The Berezin Calculus, University of Florida, Preprint (1997).

[9] Sato, M., Miwa, T. and Jimbo, M., Holonomic quantum fields I-V, Publ. RIMS, Kyoto Unıv., 14 (1978), 223-267, 15 (1979), 201-278, 15 (1979), 577-629, 15 (1979), 871972, 16 (1980), 531-584.

[10] Shale, D. and Stinespring, W. F., Spinor Representations of Infinite Orthogonal Groups, J. Math. Mech., 14 (1965), 315-322. 
